\title{
Dynamic evolution of cervical cancer mutations during chemoradiation using novel sampling approach
}

Bhavana V. Chapman ${ }^{1}$, Tatiana Karpinets ${ }^{3}$, Travis T. Sims ${ }^{2}$, Greyson Biegert ${ }^{1}$, Xiaogang $\mathrm{Wu}^{3}$, Andrea Y. Delgado Medrano ${ }^{1}$, Patricia J. Eifel ${ }^{1}$, Anuja Jhingran ${ }^{1}$, Lilie L. Lin $^{1}$, Lois M. Ramondetta ${ }^{2}$, Andrew M. Futreal ${ }^{3}$, Amir A. Jazaeri², Michael Frumovitz², Kathleen M. Schmeler², Jingyan Yue ${ }^{4}$, Aparna Mitra ${ }^{1}$, Kyoko Yoshida-Court' ${ }^{1}$, Travis Solley ${ }^{1}$, Geena Mathew ${ }^{1}$, Mustapha Ahmed-Kaddar ${ }^{1}$, Jianhua Zhang ${ }^{3}$, Ann H. Klopp ${ }^{1 *}$, Lauren E. Colbert ${ }^{1 *}$

${ }^{1}$ Department of Radiation Oncology, The University of Texas MD Anderson Cancer Center, Houston, TX, United States, ${ }^{2}$ Department of Gynecologic Oncology and Reproductive Medicine, Division of Surgery, The University of Texas MD Anderson Cancer Center, Houston, TX, United States, ${ }^{3}$ Department of Genomic Medicine, The University of Texas MD Anderson Cancer Center, Houston, TX, United States, ${ }^{4}$ McGovern Medical School at UTHealth, Houston, TX, United States

*Shared Corresponding Authorship

Corresponding Authors:

Lauren E. Colbert, MD MSCR

1515 Holcombe Blvd.

Houston, TX 77030

(832) $750-5112$

Icolbert@mdanderson.org
Ann H. Klopp, MD PhD

1515 Holcombe Blvd.

Houston, TX 77030

(713) 563-2444

aklopp@mdanderson.org 
3 Running Title: Mutations during CRT for cervical cancer

4

5 Keywords: cervical cancer, chemoradiation, whole exome sequencing, tumor mutation

6 burden,

8 Word Count: 4396

9 Tables: 1

10 Figures: 5

11 Supplemental Tables/Figures: 7

13 Funding

14 This study was partially funded by the Radiological Society of North America (RSNA)

15 Resident/Fellow Award (LEC), National Institutes of Health (NIH) through MD

16 Anderson's Cancer Center Support Grant P30CA016672 and the NIH T32 grant \#5T32

17 CA101642-14 (TTS) and The University of Texas MD Anderson Cancer Center HPV-

18 related Cancers Moonshot (LEC, AHK).

19

\section{Conflicts of interest}

21 The authors declare no potential conflicts of interest.

\section{Statement of Translational Relevance}

24 There are no established biomarkers to predict chemoradiation (CRT) response for 25 cervical cancer patients. Serial biopsies cannot be performed due to risks of bleeding and 
fistula. We used a novel non-invasive swab-based biopsy technique to obtain serial

27 samples from a cohort of twenty-seven patients through the course of treatment, and

28 validated this approach to obtain whole exome sequencing data. We analyzed dynamic

29 tumor mutation profiles during CRT. Results from this study show that mutations in

CDK4/CDK6/cyclin D1-related genes increased at the end of CRT, suggesting this pathway as a potential driver of radiation resistance.

\section{Abstract}

34 Objective: The aim of this study was to validate a whole exome sequencing approach to longitudinally characterize the tumor mutational profile of cervical cancer patients undergoing chemoradiation (CRT). Experimental Design: Cervical cancer tumor specimens from twenty-seven patients undergoing chemoradiation were collected before and throughout CRT and whole exome sequencing (WES) was performed to characterize individual mutations and alterations in unique genes. WES data were analyzed from cervical cancer patients in The Cancer Genome Atlas (TCGA) as a comparison group. Results: Over $93 \%$ of mutated genes detected at baseline were present in TCGA. Tumor purity from collected swabs correlated with MRI tumor volumes during the course of

43 treatment $\left(R^{2}=0.969\right)$. CDK4/CDK6/cyclin D1-related gene mutations involved in the

44 ERK1/2, p16INK4, and p53 pathway and G1/S checkpoint most commonly persisted at

45 the end of CRT. Conclusion: This non-invasive swab technique to serially sample tumor during CRT will allow new discoveries of dynamic tumor mutational profile changes during

47 chemoradiation for mucosal tumors. Mutations that survived or increased during the initial 
48 weeks of radiation treatment are potential drivers of radiation resistance including the

49 CDL4/CDK6/cyclin D1-related pathway.

\section{Introduction}

52 Cervical cancer continues to be one of the most common gynecologic cancers among 53 women globally. ${ }^{1}$ Approximately 13,000 new cases of invasive cervical cancer will be

54 diagnosed in the United States in 2019 , resulting in more than 4,000 deaths. $^{2}$ It is well

55 established that persistent exposure to high-risk human papilloma viruses (HPVs) plays an essential role in cervical dysplasia and carcinogenesis, causing the vast majority of

57 cervical cancer. ${ }^{3}$ The global burden of cervical cancer is growing despite the development 58 of HPV vaccines aimed at preventing the disease. Vaccines currently have a low adoption

59 rate, and it will undoubtedly be decades before cervical cancer rates are significantly 60 reduced. ${ }^{4}$ Consequently, multimodality therapy with chemoradiation (CRT) continues to 61 be the standard of care for treating locally advanced disease. ${ }^{5}$ As a result, novel 62 therapeutic targets directed at cervical cancer could expand upon current strategies to combat this gynecologic malignancy.

65 Past studies have identified somatic mutations in PIK3CA, PTEN, TP53, and KRAS in 66 cervical carcinoma. ${ }^{6-8}$ But in recent years, next generation sequencing has notably 67 increased our understanding of cervical cancer biology. 9,10 Whole exome sequencing 68 (WES) on cervical cancer samples provides complementary, pathway-generating insight 69 on somatic mutations, mutational burden, and single nucleotide human exome variants 70 (SNVs) that may be functionally significant. ${ }^{9}$ Several studies have evaluated these 
71 genomic alterations in cervical cancer. In cervical squamous cell carcinoma, somatic

72 mutations include recurrent $E 322 K$ substitutions in the MAPK1 gene, inactivating

73 mutations in the HLA-B gene, and mutations in EP300, FBXW7, NFE2L2, TP53 and

74 ERBB2. ${ }^{9}$ The Cancer Genome Atlas (TCGA) project identified SHKBP1, ERBB3, CASP8,

$75 \quad$ HLA-A and TGFBR2 as novel significantly mutated genes. ${ }^{10}$ Another study used WES to

76 identify 64 somatic mutation genes in 3 cervical tumors and found that the HPV16-positive

77 tumors had fewer somatic mutated genes than HPV-negative tumors, which was

78 validated in the TCGA dataset. ${ }^{11}$ In cervical small cell neuroendocrine tumors, ATRX,

$79 E R B B 4$, and genes in the Akt/mTOR pathway were most frequently mutated on WES. ${ }^{12}$

80 Though recent studies have recognized mutation prevalence and a variety of gene

81 expression signatures, none of the studies to date have conducted WES on longitudinal

82 tumor samples over the course of treatment. It remains unclear what mutational

83 differences may predict and distinguish treatment response.

85 Here we report serial whole exome sequencing of 27 locally advanced cervical cancer

86 tumors. Our goal was to longitudinally characterize the mutational profile of cervical

87 cancer samples from a cohort of patients undergoing CRT. We hypothesized that

88 mutations present at baseline may change in real time throughout the course of CRT.

89 Previously, serial biopsies during CRT have been impossible to obtain due to risks of

90 bleeding and fistula, but our novel non-invasive swab-based biopsy technique allows us

91 to serially sample and analyze the tumor mutational profile during the course of treatment.

92 The goal of this study was to validate the use of this non-invasive technique to 
93 demonstrate serial changes in mutations, and, in turn, identify potential mutations

94 associated with therapeutic resistance.

96 Materials and Methods

$97 \quad$ Patient population and treatment characteristics

98 Patients were enrolled in an IRB approved (2014-0543) multi-institutional prospective

99 clinical trial at The University of Texas MD Anderson Cancer Center and the Harris Health

100 System, Lyndon B. Johnson General Hospital Oncology Clinic (Figure 1A). This study

101 was designed to evaluate metagenomic changes in the cervical and intestinal microbiota

102 during chemoradiation. Inclusion criteria were newly diagnosed locally advanced cervical

103 cancer per the Federation of Gynecology and Obstetrics (FIGO) 2009 staging system.

104 Patients with clinical stage IB1-IVA, visible, exophytic tumor on speculum examination

105 with planned treatment of intact cervical cancer with definitive radiation therapy, including

106 external beam radiation therapy and brachytherapy with concurrent cisplatin were

107 included. Patients with any previous pelvic radiation therapy or treatment for cervical

108 cancer, including transvaginal cone irradiation, were excluded.

110 Patients underwent the standard-of-care pretreatment evaluation for their disease,

111 including a tumor biopsy to confirm diagnosis; pelvic magnetic resonance imaging (MRI)

112 and positron emission tomography/computed tomography (PET/CT); and standard 113 laboratory evaluations, including a complete blood cell count, measurement of

114 electrolytes, and evaluation of renal and liver function. HPV-positivity was confirmed by 115 p16 staining of tumor biopsies on initial institutional pathologic review. Patients received 
116 pelvic radiation therapy to a total dose of 40 to 45 Gy delivered in daily fractions of 1.8 to

1172 Gy over 4 to 5 weeks. Following completion of pelvic radiation therapy, patients received

118 intracavitary brachytherapy with pulsed-dose or high-dose rate treatments over 44 to 48

119 hours or the equivalent with high-dose-rate brachytherapy. Patients received cisplatin 40

$120 \mathrm{mg} / \mathrm{m}^{2}$ weekly delivered according to standard institutional protocol. Patients underwent

121 repeat $\mathrm{MRI}$ at the completion of external beam radiation therapy or at the time of 122 brachytherapy, as indicated by the extent of disease.

124 Sample collection and DNA extraction

125 Isohelix swabs (product \# DSK-50 and XME-50, www.isohelix.com, UKSamples) were 126 brushed against the viable cervical tumor several times by an attending radiation 127 oncologist or gynecologic oncologist at either The University of Texas MD Anderson 128 Cancer Center or Harris Health, Lyndon B. Johnson Hospital. The isohelix swab has a 129 unique matrix design that permits the yield of one to five mcg of high quality DNA sufficient 130 for sequencing applications from a single swab of the tumor surface ${ }^{13}$. Patients underwent 131 swabs at baseline, the end of week 1 (after 5 fractions), at the end of week 3 (after 10-15 132 fractions), and within a week prior to the first brachytherapy treatment or at the time of 133 brachytherapy (week 5), for a total of four swabs during radiation therapy. Attention was 134 taken to serially swab the same general tumor region. DNA was extracted from normal 135 buccal and cervical cancer samples per Isohelix \# DSK-50 manufacturer's instructions. 
138 The Illumina WES assay was optimized for normal buccal control and cervical tumor DNA 139 swab samples. Captured libraries were sequenced on a HiSeq 2000 series (Illumina Inc., 140 San Diego, CA, USA) on a TruSeq v3 Paired-end Flowcell according to manufacturer's 141 instructions at a cluster density between $700-1000 \mathrm{~K}$ clusters $/ \mathrm{mm} 2$. Sequencing was 142 performed on a HiSeq 2000 series for $2 \times 100$ paired end reads with a 7 nucleotide read 143 for indexes using Cycle Sequencing v3 reagents (Illumina). All regions were covered by $144>200$ reads.

WES exome sequencing data were aligned to human reference genome using BWA. ${ }^{14}$

147 Duplicate reads were removed using Picard and then realigned and recalibrated by the 148 Genome Analysis Toolkit. ${ }^{15}$ Samples used for each analysis are outlined in Figure 1B. 149 Tumor purity was calculated from single nucleotide variants by TPES. ${ }^{16}$ MuTect and 150 Pindel were applied to detect somatic SNVs and small InDels using buccal DNA samples 151 from each patient as normal controls. ${ }^{17,18}$ Variants were classified into 3 categories: 152 somatic, germline, and loss of heterozygosity based on variant allele frequencies in the 153 tumor and matched normal buccal samples. Somatic copy number alterations reporting 154 gain or loss of each exon were noted using a previously described algorithm. ${ }^{19}$ COSMIC, 155 TCGA, and dbSNP databases were used to identify potential functional consequences 156 of detected variants which were annotated using VEP, Annovar, CanDrA. ${ }^{20-23}$

158 Cervical tumors were contoured on serial MR imaging for six patients during the course 159 of CRT using RayStation. 3-dimensional volumes were generated from contours. 
160 Regression analysis was utilized to evaluate the goodness of fit between tumor purity 161 (previously described) versus MRI tumor volumes.

163 Comparison against published databases

164 TCGA mutations were downloaded using the TCGABiolinks package in R. The mutect 165 and indel pipeline output of the most recent version of the "CESC" dataset was used. The $166 \mathrm{NCl}$ Genomic Data Commons (GDC) Data Portal was also used to query submitted 167 cervical cancer samples with available mutation data.

$\underline{\text { Pathway enrichment analysis }}$

170 To query for potential radiation-related genes, the list of altered genes in all patient 171 samples was queried using the Clarivate Analytics MetaCore database.

173 Selection of potential drivers of radiation resistance

174 The mutations and specific genes that had the greatest percent change from baseline in an individual patient were summed to obtain an average change in the population. These

176 mutations and genes were selected as potential drivers of radiation resistance and 177 pathway analysis was performed using MetaCore database.

179 Data Sharing

180 All code used for these analyses and de-identified raw datasets of mutations will be 181 shared at github.com/ColbertLab/Whole-Exome-Seq-Paper. 
183

184

Baseline somatic mutational landscape

The distribution of detected mutations for each patient across the 4 timepoints is shown in Figure 2A, while detected mutations for each patient with respect to each timepoint is shown in Figure 2B. The majority of baseline mutations were nonsynonymous. In evaluating exonic mutations across timepoints, nonsynonyous mutations were more prevalent in tumor samples whereas stopgain and stoploss mutations were less abundant (Figure 2C). Breakpoints of large deletions and medium-sized insertions from 
paired-end short reads were used as a means to detect frameshift/nonframeshift and insertions/deletions (Figure 2D).

All substitutions, insertions and deletions were filtered for exonic, nonsynonymous stop/gain and stop/loss alterations. The entire list of mutations is available in supplemental table 1. This overall landscape was very similar to known TCGA mutations

211 in the most recent version of the CESC dataset (Figure 3). Overall, 94\% (1339/1430)

212 detected exonic, nonsynonymous, stop/gain or stop/loss gene alterations in our samples 213 were present in the TCGA CESC dataset (Figure 3A). Supplemental table 2 lists all 214 overlapping altered genes between TCGA CESC and our samples overall and at each 215 individual timepoint. Supplemental table 3 and supplementary table 4 list the 216 frequencies of all gene alterations in TCGA (Figure 3B) and our sample dataset (Figure $2173 \mathrm{C}$ ), ranked by frequency. Among the top 30 most frequently mutated genes, changes in $218 T T N$, MUC4, KMT2C, LRP1N, PIK3CA, and KMT2C and KMT2D were present in both 219 groups. There were some unique alterations to our population, including $C D C 27$, an 220 important gene for radiation response. Baseline pathway enrichment analysis using 221 ranking of the most common mutations indicates that mutations involved in the PI3K/Akt and Wnt/beta-catenin pathways were commonly detected, consistent with known cervical cancer mutations (Figure 3D; supplementary table 5).

Evolution of tumor mutational changes

226 For patients who had adequate DNA at all timepoints, baseline exonic, non-synonymous, 227 stop/gain and stop/ loss mutations were filtered, and changes in frequency of these 
228 alterations over time was plotted (Figure 4A), demonstrating that overall, mutations 229 present at baseline decrease over time, although some persist. Ranking of altered genes

230 changed from baseline (Figure 4B) to week1 (4C), week 3 (4D) and week 5 (4E), 231 suggesting that there is dynamic evolution throughout chemoradiation. As a whole, 232 mutations in MUC4 persisted throughout the course of chemoradiation.

234 Changes in MRI tumor volumes and mutational profile in a patient with persistent tumor

235 Given this non-invasive biopsy technique, in order to quantify changes in allele

236 frequency for low quality DNA, we compared calculated tumor purities to known tumor

237 volume at each time point. Figure 5A demonstrates serial MRI images taken at

238 baseline and week 5 for one poor responder, with tumor contoured in gray. 3-

239 dimensional tumor volumes were calculated at each timepoint for the 6 patients with

240 serial MRl's. There was a high degree of linear correlation (Figure 5B, $R^{2}=0.969$ )

241 between computed tumor purity and known tumor volume. Once this was confirmed,

242 calculated percent change in allele frequencies of individual mutations over time were

243 calculated (supplemental table 6). All significantly changed mutations in individual

244 patients were then summed and averaged to determine changes in individual mutations

245 for the whole population (Figure 5C; supplemental table 7). The top 20 ranked genes

246 by change at week 5 included TCF25, MUC2, PARP10 and PIK3CA (Figure 5D).

247 Pathway analysis of the ranked mutated genes present at week 5 demonstrate

248 perturbations in the G1/S transition via CDK4/CDK6/cyclin D1-related gene mutations, a 249 key radiation sensitivity pathway (Figure 5E). 


\section{Discussion}

252

253

254

255

256

257

258

259

260

261

262

263

264

265

266

267

268

269

270

271

272

273

To our knowledge, this is the first study to serially sample cervical cancer tumors and comprehensively characterize alterations in the mutational landscape using whole exome sequencing during the course of CRT. Obtaining tumor biopsies during the course of treatment is painful for patients and logistically challenging. Historically, the aforementioned limitations pose significant barriers to evaluating dynamic changes in tumor genetic signatures in response to ongoing treatment. Our novel, non-invasive swab-based biopsy technique which allows for DNA collection from cervical brushings during pelvic exam circumvents these obstacles. We noted that tumor purity correlated with residual cervical tumor volumes measured from MRI performed at corresponding timepoints. Moving forward, this method could be applied to other gynecologic, head and neck, anorectal, and skin malignancies to establish biomarker predictors for treatment response and potential drivers of treatment resistance which may be undetectable from the analysis of initial biopsies only.

The distribution of somatic mutations detected in our patient cohort is similar to other patient cohorts which further validates our findings. There was a high degree of overlap between the mutated genes detected at baseline in our patient samples and the TCGA cervical cancer cohort which is comprised of chemoradiation-naive patients. MUC4, the most frequently mutated gene in our cohort, encodes a membrane-bound mucin with promotes tumorigenicity through epithelial invasion, immune surveillance evasion, and suppression of apoptosis. ${ }^{25}$ It is implicated in cervical squamous dysplasia and carcinoma. ${ }^{26}$ MUC4 expression was found to be inversely related to survival in patients with epithelial carcinomas. ${ }^{27}$ MUC16, another mucin membrane protein, encodes the 
274 extracellular domain of CA125 and is commonly mutated in ovarian and gastric cancer. ${ }^{32}$

275 MUC16 is implicated in aberrant cell proliferation via the JAK-STAT pathway and EGFR

276 signaling with activation of downstream Akt and ERK1/2. ${ }^{33} T T N$, another frequently

277 mutated gene detected in our cohort and TCGA, encodes for Titin, the largest protein

278 found in humans encoded by 364 exons. ${ }^{28}$ Mutations in TTN have been reported in dilated

279 cardiomyopathy and Titin autoantibodies have been detected in patients with Sjögren's

280 syndrome and systemic scleroderma. ${ }^{29,30}$ Patients with mutations in TTN have been

281 shown to possess higher tumor mutational burden and improved response to immune

282 checkpoint blockade therapy in various solid tumors of skin, lung, stomach compared to

283 those with wild-type TTN. ${ }^{31}$

284 Pathway analysis of baseline mutations in our cohort demonstrates that the MAPK

285 mitogenic pathway was the most commonly perturbed pathway overall. Phosphoinositide

286 3-kinase (PI3K) pathway-related mutations were also prevalent. Others have reported

287 perturbations in PIK3CA, an oncogene part of the ERBB2/PI3K/AKT/mTOR pathway with

288 a battery of druggable targets, present in over $50 \%$ of cervical tumor and cervical cancer

289 cell lines, which is consistent with our findings. ${ }^{3}$ Schuurbiers et al. previously described

290 PI3K-Akt pathway activation and mechanisms of radiation resistance in non-small cell

291 lung cancer which could similarly be involved in cervical cancer. ${ }^{35}$ Additionally, in vitro

292 studies demonstrate the PIK3CA E545K mutation results in resistance to platinum

293 chemotherapy. ${ }^{36}$ The E545K mutation is the single most common mutation among TCGA

294 cervical cancer patients and is present at a rate of $13 \%(38 / 289)$.

295 When examining mutations that persisted at the end of radiation, those involved in the 296 G1/S transition involving ERK1/2, p16INK4, and p53 were most frequently mutated and 
297 driven by several CDK4/CDK6/cyclin D1-related gene mutations. The G1/S checkpoint is

298 involved in the DNA damage repair process and radiation-induced cell death. ${ }^{37}$ The

299 aberrant CDK4/CDK6/cyclin D1 pathway is well known in the pathogenesis of HPV300 associated malignancies and CDK4/6 inhibitors are even being explored as potential 301 therapeutic agents in the treatment of HPV-negative cervical cancer. ${ }^{38,39}$

Presently, there is a paucity of data examining serial genomic profiling of tumors before and after treatment, especially in the locally advanced setting. In the TCGA cervical cancer cohort, over $60 \%(138 / 228)$ patients had FIGO stage I disease, and over $75 \%$ $(106 / 138)$ of which were treated with upfront hysterectomy. ${ }^{10}$ The majority of these early stage cervical cancer patients likely did not require adjuvant therapy. Thus, the baseline mutational profile of patients from TCGA may not be directly comparable to patients with locally advanced cervical cancer who go on to receive definitive chemoradiation as described in our cohort. WES feasibility studies have been described in the recurrent and metastatic setting, but studies examining de novo, disease site-specific populations are

311 limited. ${ }^{40} \mathrm{~A}$ recent series of 28 patients with rectal cancer reported WES analysis before 312 and after preoperative CRT demonstrated persistent mutations in CTDSP2, APC, KRAS, TP53 and NFKBIZ conferred treatment resistance. ${ }^{41}$ Next-generation sequencing has

314 been described to identify gene signatures predictive of treatment response from initial 315 biopsies and surgical specimens after preoperative radiotherapy for early stage breast 316 cancer (PMID31525407).

317 In conclusion, this study provides evidence that a novel swab-based technique can be 318 utilized to serially sample cervical tumors for whole exome sequencing analysis. We posit 319 that mutations that survive the initial weeks of radiation treatment may be clinically 
320 relevant drivers of radiation resistance. Future work will focus on characterizing the clonal

321 architecture of residual tumors to identify granular molecular signatures predictive of

322 treatment response.

323 Conflicts of Interest: The authors report no conflicts of interest, financial or otherwise,

324 related to the subject matter of the article submitted.

325

\section{Author Contribution}

327 All authors were involved with subject identification and data collection, interpretation of

328 statistical analysis, and review and approval of final manuscript. Study concept was 329 developed by AHK, LEC and BVC. Analyses were performed by LEC, THK, XW, GB. 330 Drafting and editing of manuscript was performed by all authors.

\section{Acknowledgements}

333 This work was supported in part by the Radiological Society of North America (RSNA)

334 Resident/Fellow Award (LEC), National Institutes of Health (NIH) through MD Anderson's

335 Cancer Center Support Grant P30CA016672 and the NIH T32 grant \#5T32 CA101642-

33614 (TTS) and The University of Texas MD Anderson Cancer Center HPV-related Cancers

337 Moonshot (LEC, AHK). The human subjects who participated in this study are gratefully 338 acknowledged, in addition to our clinical research team, and scientific publications team.

\section{References}

341 1. Siegel, R. L., Miller, K. D. \& Jemal, A. Cancer statistics, 2019. CA. Cancer J. Clin. 69, 7-34 
2. Islami, F., Fedewa, S. A. \& Jemal, A. Trends in cervical cancer incidence rates by age, race/ethnicity, histological subtype, and stage at diagnosis in the United States. Prev. Med. 123, 316-323 (2019).

3. zur Hausen, H. Papillomaviruses in the causation of human cancers - a brief historical account. Virology 384, 260-265 (2009).

4. Bruni, L. et al. Global estimates of human papillomavirus vaccination coverage by region and income level: a pooled analysis. Lancet Glob. Health 4, e453-463 (2016).

5. Monk, B. J., Tewari, K. S. \& Koh, W.-J. Multimodality therapy for locally advanced cervical carcinoma: state of the art and future directions. J. Clin. Oncol. Off. J. Am. Soc. Clin. Oncol. 25, 2952-2965 (2007).

6. Crook, T. et al. Clonal p53 mutation in primary cervical cancer: association with humanpapillomavirus-negative tumours. Lancet Lond. Engl. 339, 1070-1073 (1992).

7. Mclntyre, J. B. et al. PIK3CA mutational status and overall survival in patients with cervical cancer treated with radical chemoradiotherapy. Gynecol. Oncol. 128, 409-414 (2013).

8. Kang, S. et al. Inverse correlation between RASSF1A hypermethylation, KRAS and BRAF mutations in cervical adenocarcinoma. Gynecol. Oncol. 105, 662-666 (2007).

9. Ojesina, A. I. et al. Landscape of genomic alterations in cervical carcinomas. Nature 506, $371-375(2014)$.

10. Cancer Genome Atlas Research Network et al. Integrated genomic and molecular characterization of cervical cancer. Nature 543, 378-384 (2017).

11. Wu, Y. et al. Whole-exome and RNA sequencing reveal novel insights into the pathogenesis of HPV associated cervical cancer. Cancer Biomark. Sect. Dis. Markers 25, 341-350 (2019).

12. Cho, S. Y. et al. Cervical small cell neuroendocrine tumor mutation profiles via whole exome sequencing. Oncotarget 8, 8095-8104 (2017).

13. Simon, C. et al. A key role for $\mathrm{EZH} 2$ and associated genes in mouse and human adult T-cell 
acute leukemia. Genes Dev. 26, 651-656 (2012).

14. Gnirke, A. et al. Solution hybrid selection with ultra-long oligonucleotides for massively parallel targeted sequencing. Nat. Biotechnol. 27, 182-189 (2009).

15. Li, H. \& Durbin, R. Fast and accurate short read alignment with Burrows-Wheeler transform. Bioinforma. Oxf. Engl. 25, 1754-1760 (2009).

16. Locallo, A., Prandi, D., Fedrizzi, T. \& Demichelis, F. TPES: tumor purity estimation from SNVs. Bioinforma. Oxf. Engl. 35, 4433-4435 (2019).

17. Cibulskis, K. et al. Sensitive detection of somatic point mutations in impure and heterogeneous cancer samples. Nat. Biotechnol. 31, 213-219 (2013).

18. Ye, K., Schulz, M. H., Long, Q., Apweiler, R. \& Ning, Z. Pindel: a pattern growth approach to detect break points of large deletions and medium sized insertions from paired-end short reads. Bioinforma. Oxf. Engl. 25, 2865-2871 (2009).

19. Lonigro, R. J. et al. Detection of somatic copy number alterations in cancer using targeted exome capture sequencing. Neoplasia N. Y. N 13, 1019-1025 (2011).

20. Bamford, S. et al. The COSMIC (Catalogue of Somatic Mutations in Cancer) database and website. Br. J. Cancer 91, 355-358 (2004).

21. McLaren, W. et al. Deriving the consequences of genomic variants with the Ensembl API and SNP Effect Predictor. Bioinforma. Oxf. Engl. 26, 2069-2070 (2010).

22. Wang, K., Li, M. \& Hakonarson, H. ANNOVAR: functional annotation of genetic variants from high-throughput sequencing data. Nucleic Acids Res. 38, e164 (2010).

23. Mao, Y. et al. CanDrA: cancer-specific driver missense mutation annotation with optimized features. PloS One 8, e77945 (2013).

24. Miller, C. A. et al. SciClone: inferring clonal architecture and tracking the spatial and temporal patterns of tumor evolution. PLoS Comput. Biol. 10, e1003665 (2014). carcinomas. Oncotarget 8, 14147-14157 (2017). 
26. Munro, E. G. et al. Upregulation of MUC4 in cervical squamous cell carcinoma: pathologic significance. Int. J. Gynecol. Pathol. Off. J. Int. Soc. Gynecol. Pathol. 28, 127-133 (2009).

27. Lee, H. K., Cho, M.-S. \& Kim, T. H. Prognostic significance of muc4 expression in gallbladder carcinoma. World J. Surg. Oncol. 10, 224 (2012).

28. Eckels, E. C., Tapia-Rojo, R., Rivas-Pardo, J. A. \& Fernández, J. M. The Work of Titin Protein Folding as a Major Driver in Muscle Contraction. Annu. Rev. Physiol. 80, 327-351 (2018).

29. Gerull, B. et al. Mutations of TTN, encoding the giant muscle filament titin, cause familial dilated cardiomyopathy. Nat. Genet. 30, 201-204 (2002).

30. Ohyama, K. et al. Proteomic profiling of antigens in circulating immune complexes associated with each of seven autoimmune diseases. Clin. Biochem. 48, 181-185 (2015).

31. Jia, Q., Wang, J., He, N., He, J. \& Zhu, B. Titin mutation associated with responsiveness to

32. Li, X., Pasche, B., Zhang, W. \& Chen, K. Association of MUC16 Mutation With Tumor Mutation Load and Outcomes in Patients With Gastric Cancer. JAMA Oncol. 4, 1691-1698 (2018).

33. Aithal, A. et al. MUC16 as a Novel Target for Cancer Therapy. Expert Opin. Ther. Targets

$$
\text { 22, 675-686 (2018). }
$$

34. Zammataro, L. et al. Whole-exome sequencing of cervical carcinomas identifies activating

35. Schuurbiers, O. C. J. et al. The PI3-K/AKT-pathway and radiation resistance mechanisms in non-small cell lung cancer. J. Thorac. Oncol. Off. Publ. Int. Assoc. Study Lung Cancer 4, 761-767 (2009).

36. Arjumand, W. et al. Phosphatidyl inositol-3 kinase (PIK3CA) E545K mutation confers cisplatin resistance and a migratory phenotype in cervical cancer cells. Oncotarget 7, 
Chapman et al. 2019

82424-82439 (2016).

422 37. Pawlik, T. M. \& Keyomarsi, K. Role of cell cycle in mediating sensitivity to radiotherapy. Int.

423 J. Radiat. Oncol. Biol. Phys. 59, 928-942 (2004).

424 38. Munger, K., Gwin, T. K. \& McLaughlin-Drubin, M. p16 in HPV-associated cancers.

$425 \quad$ Oncotarget 4, 1864-1865 (2013).

426 39. Xiong, Y. et al. Ribociclib, a selective cyclin D kinase 4/6 inhibitor, inhibits proliferation and

427 induces apoptosis of human cervical cancer in vitro and in vivo. Biomed. Pharmacother.

428 Biomedecine Pharmacother. 112, 108602 (2019).

429 40. Beltran, H. et al. Whole-Exome Sequencing of Metastatic Cancer and Biomarkers of

430 Treatment Response. JAMA Oncol. 1, 466-474 (2015).

431 41. Yang, J. et al. Genome landscapes of rectal cancer before and after preoperative 432 chemoradiotherapy. Theranostics 9, 6856-6866 (2019). 


\begin{tabular}{|c|c|c|}
\hline Characteristic & No. of patients & $\%$ \\
\hline $\begin{array}{l}\text { Age at diagnosis, years } \\
\text { Median } \\
\text { Range }\end{array}$ & $\begin{array}{c}47.5 \\
30-72\end{array}$ & \\
\hline $\begin{array}{l}\text { Race/Ethnicity } \\
\text { African American } \\
\text { American Indian/Alaskan Native } \\
\text { Asian } \\
\text { Hispanic/Latino } \\
\text { White/Caucasian }\end{array}$ & $\begin{array}{c}2 \\
1 \\
1 \\
16 \\
7\end{array}$ & $\begin{array}{r}7.4 \\
3.7 \\
3.7 \\
59.3 \\
25.9\end{array}$ \\
\hline $\begin{array}{l}\text { Histology } \\
\text { Adenocarcinoma } \\
\text { Squamous cell carcinoma }\end{array}$ & $\begin{array}{c}8 \\
19\end{array}$ & $\begin{array}{l}30.0 \\
70.0\end{array}$ \\
\hline $\begin{array}{l}2009 \text { FIGO stage } \\
\text { IB1 } \\
\text { IB2 } \\
\text { IIA } \\
\text { IIB } \\
\text { IIIB } \\
\text { IVA }\end{array}$ & $\begin{array}{l}3 \\
5 \\
2 \\
12 \\
4 \\
1\end{array}$ & $\begin{array}{c}11.1 \\
18.5 \\
7.4 \\
44.4 \\
14.8 \\
3.7\end{array}$ \\
\hline $\begin{array}{l}\text { Tumor grade } \\
\text { I } \\
\text { II } \\
\text { III } \\
\text { Unknown }\end{array}$ & $\begin{array}{l}2 \\
11 \\
9 \\
5\end{array}$ & $\begin{array}{r}7.4 \\
40.7 \\
33.3 \\
18.5\end{array}$ \\
\hline $\begin{array}{l}\text { Highest clinically involved nodal level on PET or CT } \\
\text { Para-aortic } \\
\text { Common iliac } \\
\text { External iliac } \\
\text { Internal iliac } \\
\text { Node-negative }\end{array}$ & $\begin{array}{c}4 \\
3 \\
10 \\
2 \\
8\end{array}$ & $\begin{array}{r}14.8 \\
11.1 \\
37.0 \\
7.4 \\
29.6\end{array}$ \\
\hline $\begin{array}{l}\text { Max tumor dimension on MRI, cm } \\
\text { Median } \\
\text { Range } \\
\text { Unknown }\end{array}$ & $\begin{array}{c}4.6 \\
2.2-11.5 \\
1\end{array}$ & \\
\hline $\begin{array}{l}\text { HPV genotype } \\
\text { HPV16 } \\
\text { HPV18 } \\
\text { Other high-risk HPV } \\
\text { Unknown }\end{array}$ & $\begin{array}{l}11 \\
2 \\
7 \\
7\end{array}$ & $\begin{array}{r}40.7 \\
7.4 \\
25.9 \\
25.9\end{array}$ \\
\hline $\begin{array}{l}\text { Smoking status } \\
\text { Current } \\
\text { Former } \\
\text { Never }\end{array}$ & $\begin{array}{c}3 \\
12 \\
12\end{array}$ & $\begin{array}{l}11.1 \\
44.4 \\
44.4\end{array}$ \\
\hline
\end{tabular}


A

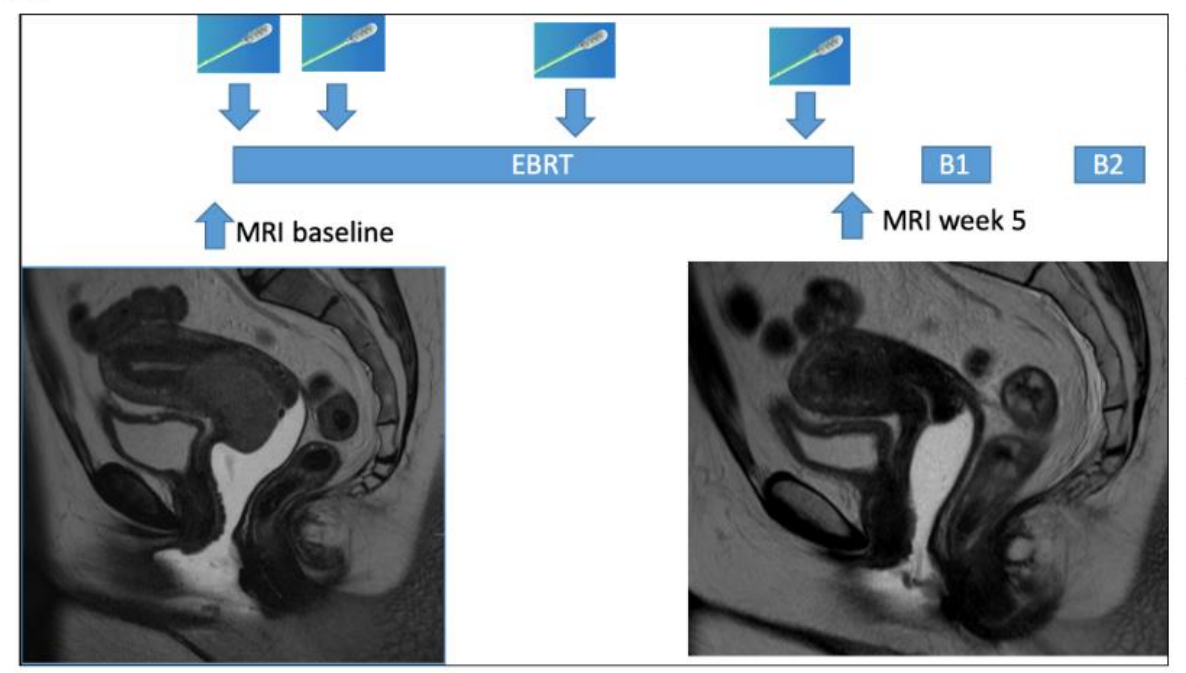

B

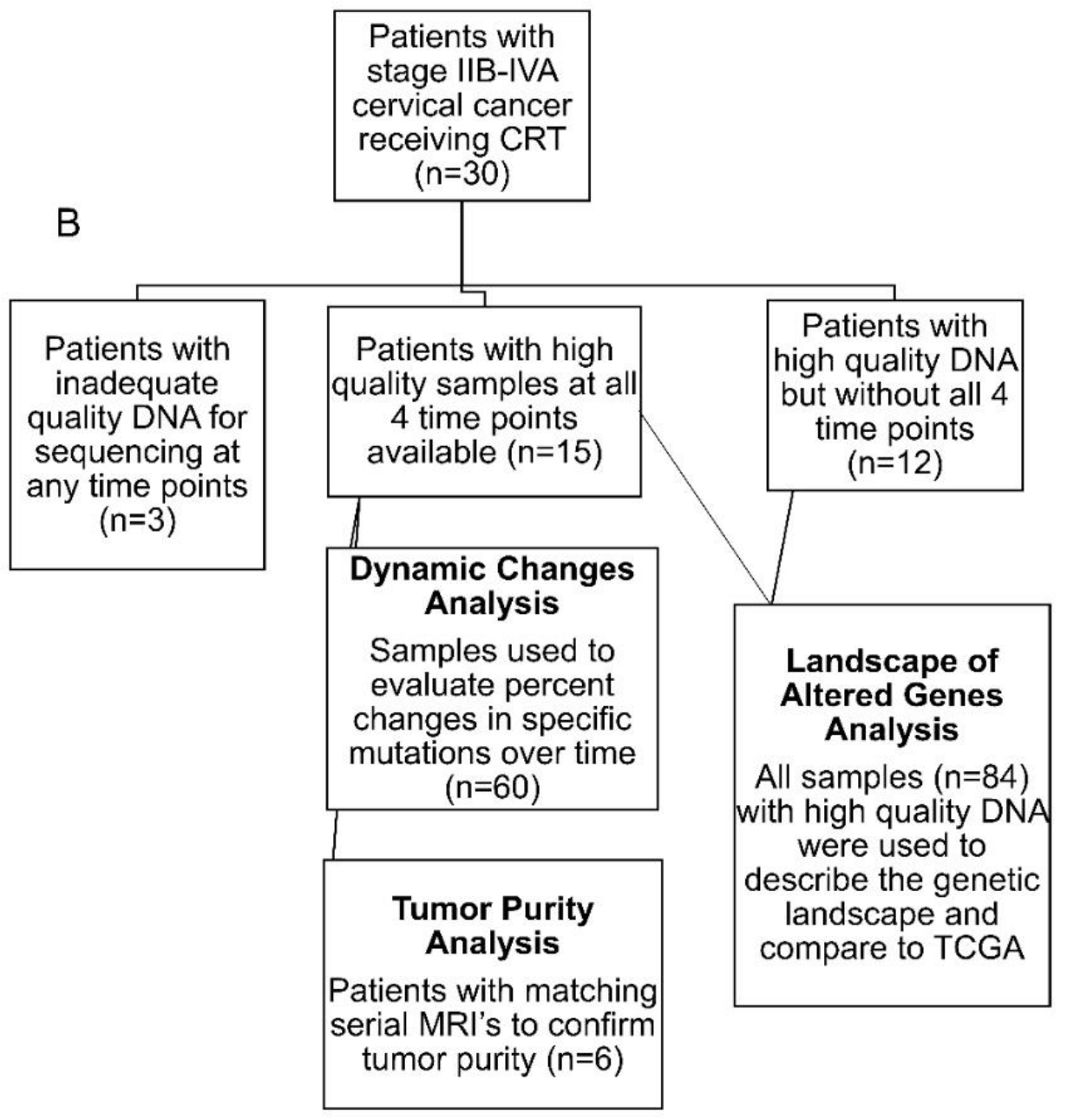

Figure 1. Overall study schema for patient sample collection and analysis. (A) Patients with locally advanced cervical cancer undergo 5 weeks of external beam radiation therapy (EBRT) followed by two brachytherapy treatments (B1 and B2), with samples collected at Baseline, Week 1, Week 3 and Week 5 of radiation therapy. Patients also underwent optional MRI imaging at baseline and at week 5. (B) 30 patients were accrued on protocol, and of these, 27 patients with 85 total samples had adequate DNA quantity and quality for sequencing. These samples were used to describe the overall mutational landscape of the population. 15 patients had samples available at all 4 timepoints, and these samples were used to analyze dynamic changes over time. 6 patients had matching samples at all timepoints and serial MRl's, and these samples were used to validate the sequencing technique using tumor purity analysis. 
A

Distribution of All Altered Genes

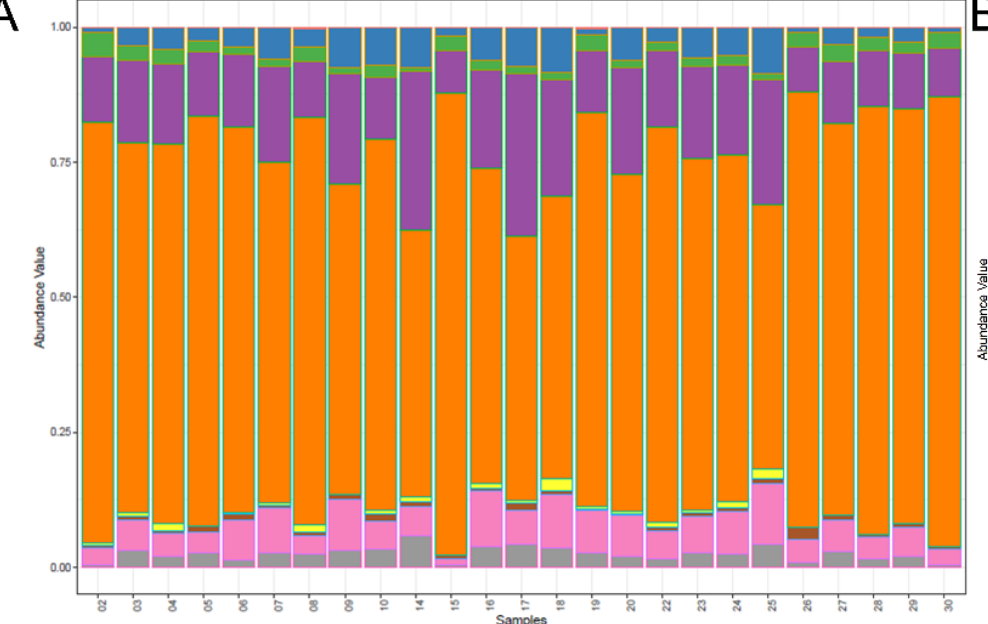

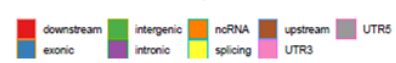

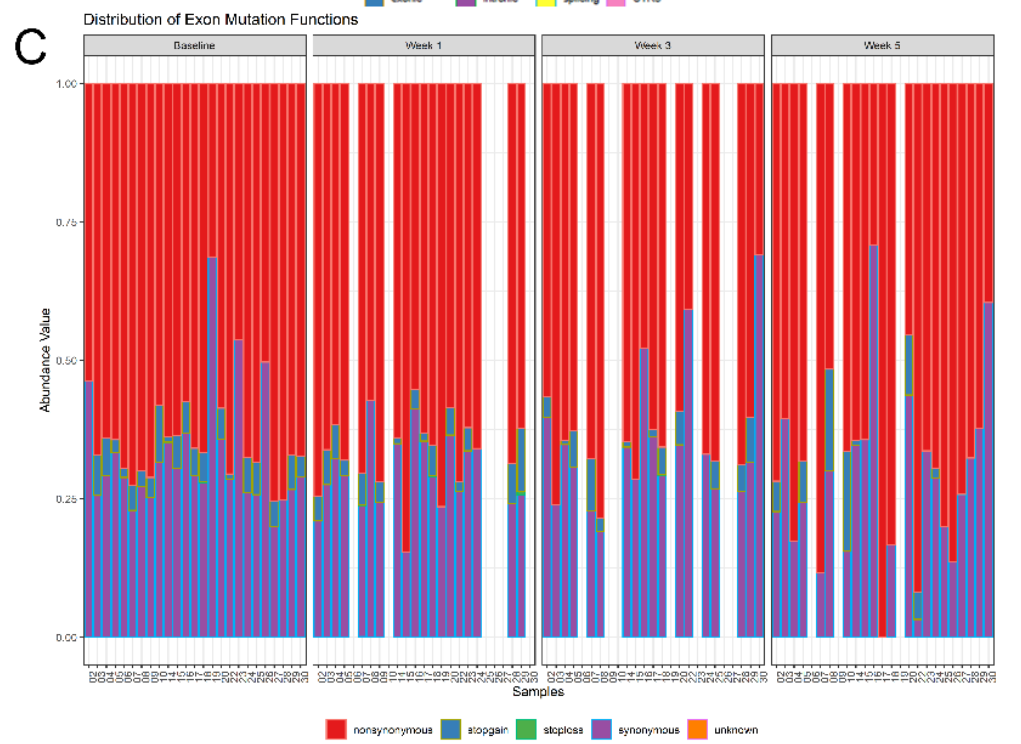

Distribution of Mutation Functions

$\mathrm{B}$

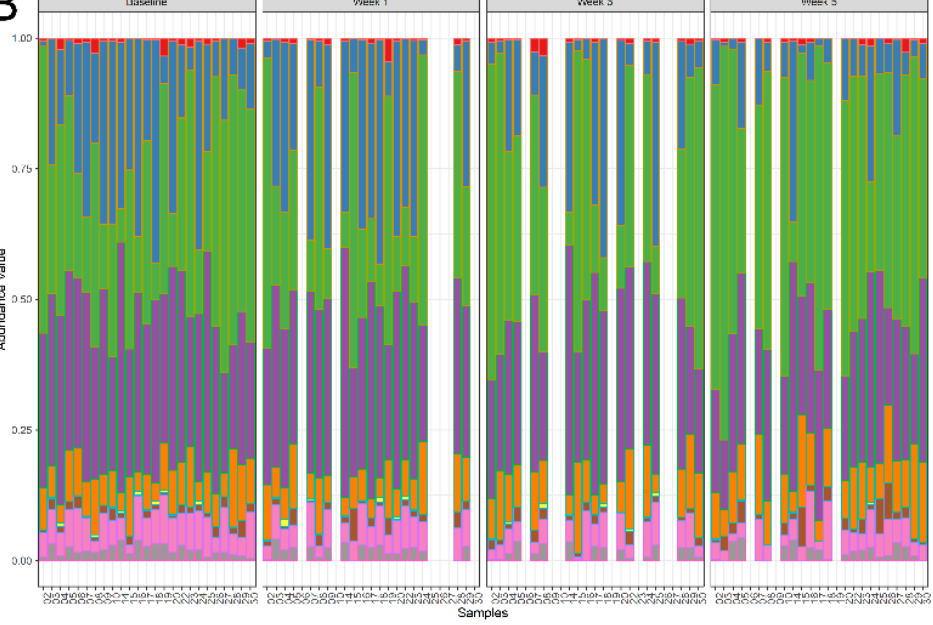

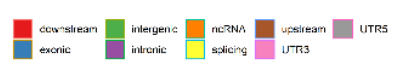

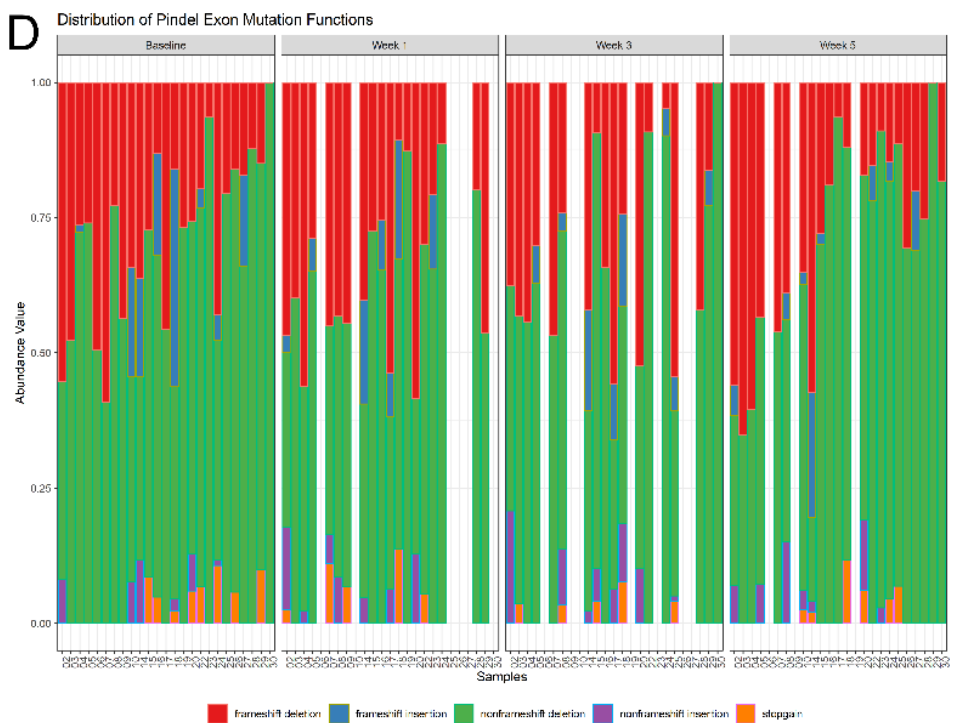

Figure 2. Baseline mutation type stacked bar plots (TCGAvs our population).

(A) Stacked bar chart displaying the relative abundance of mutation events in all genes for each patient across all available samples throughout treatment. Similar to (A), (B) highlights the relative abundance of mutation events in all genes for each patient at separate timepoints during treatment. Thus showing that exon mutations as critical contributors to the mutation burden. Figure (C) then displays the relative distribution of exon specific mutation functions for each patient, at separate timepoints during treatment. Additionally, figure (D) shows the relative abundance of insertion/deletion mutations present in exons for each patient stratified by time point. For figures BD, patient samples with missing data are represented by empty bars. 


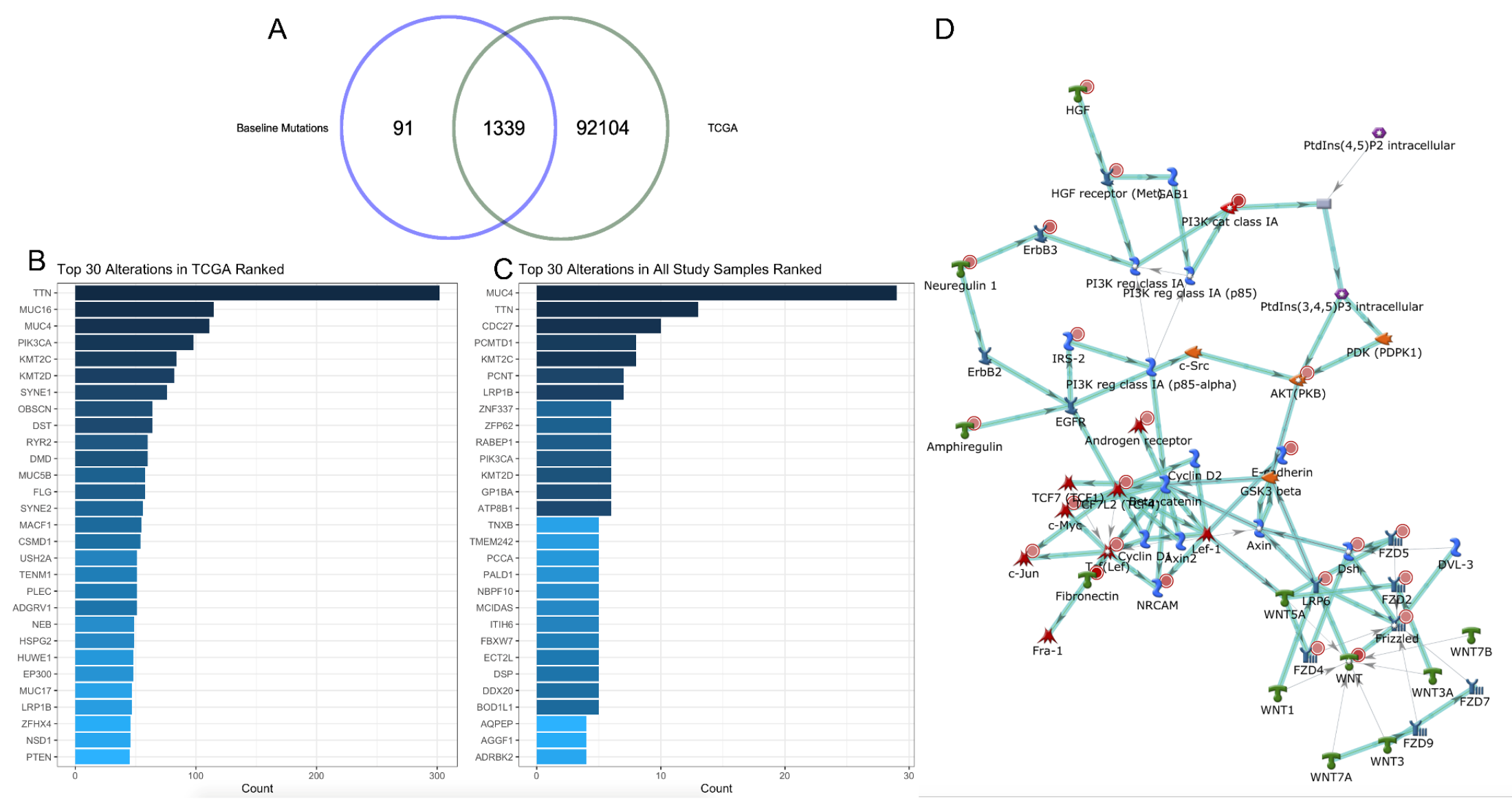

Figure 3. Overall Gene Alterations from All Samples is similar to landscape of TCGA cervical squamous cell carcinoma dataset. (A) $94 \%$ of (1339/1430) altered genes in baseline samples (defined as substitutions, insertions or deletions in gene), were also identified in the TCGA dataset, suggesting accurate identification of cervical cancer related genes. (B) Distribution of top 30 most altered genes in our study sample and TCGA (C) is also similar. (D) Pathway analysis was performed of top 100 altered genes in the study samples at baseline, and the most frequency pathway is shown here, centered on PI3K and related genes, again consistent with expected mutational landscape for cervical cancer. 

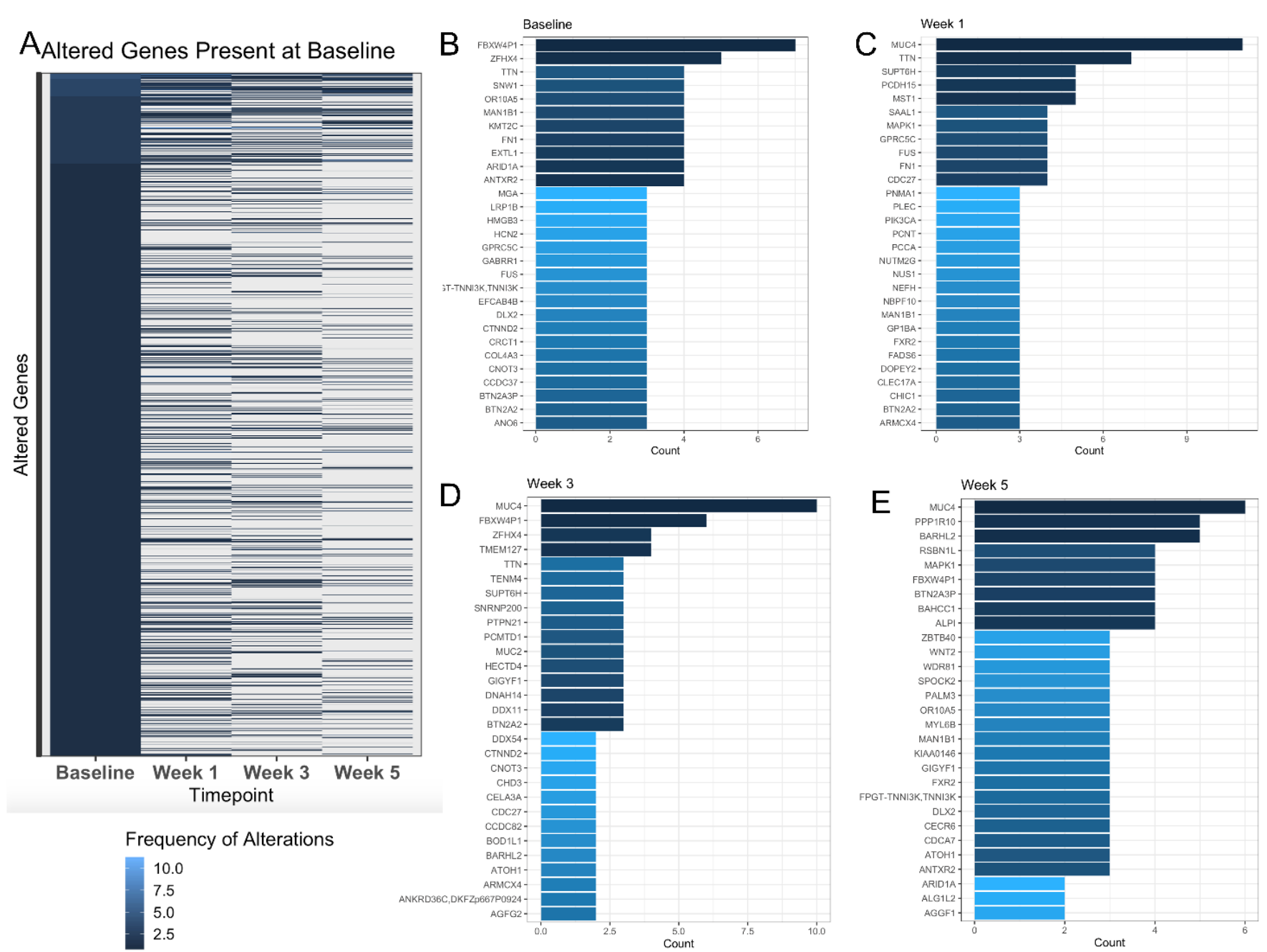

Figure 4. Changes in gene alterations over time for patients with all 4 time points $(N=15)$ demonstrated overall decrease over time, but shifts in specific alterations. (A) All altered genes (exonic non-synonymous mutations, including substitutions, insertions and deletions) present at baseline decrease throughout the course of CRT. Alterations present at Baseline (B), Week 1 (C), Week3 (D), and Week 5 (E) 
A
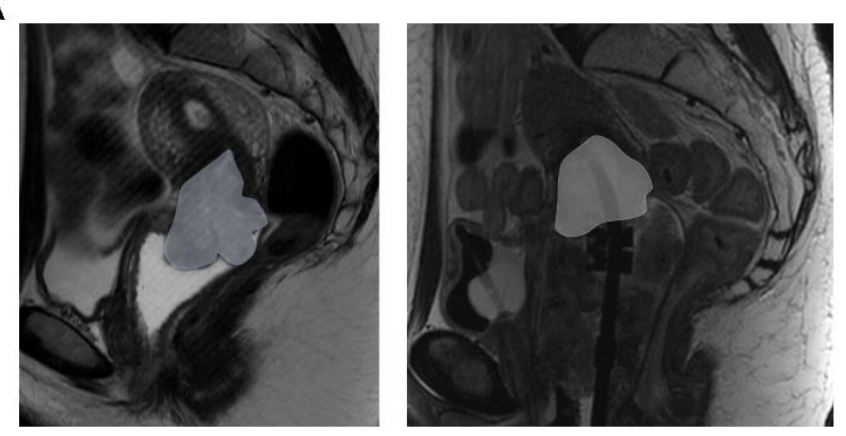

B

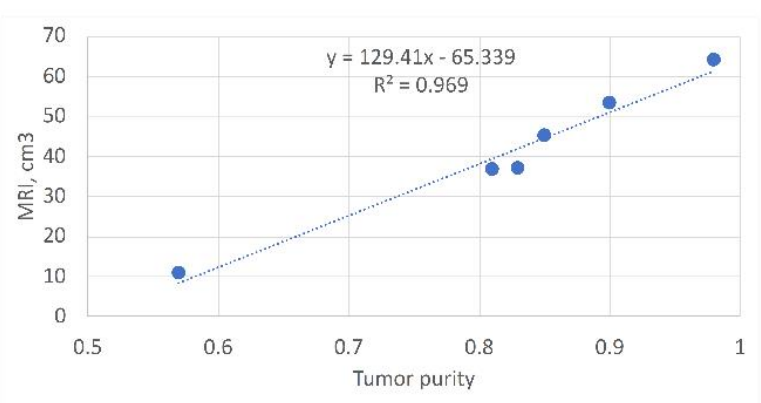

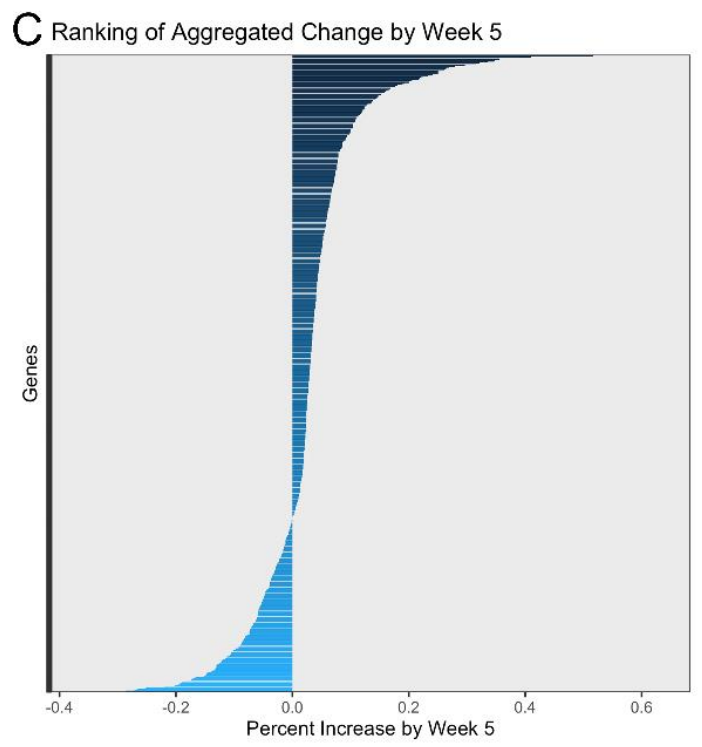

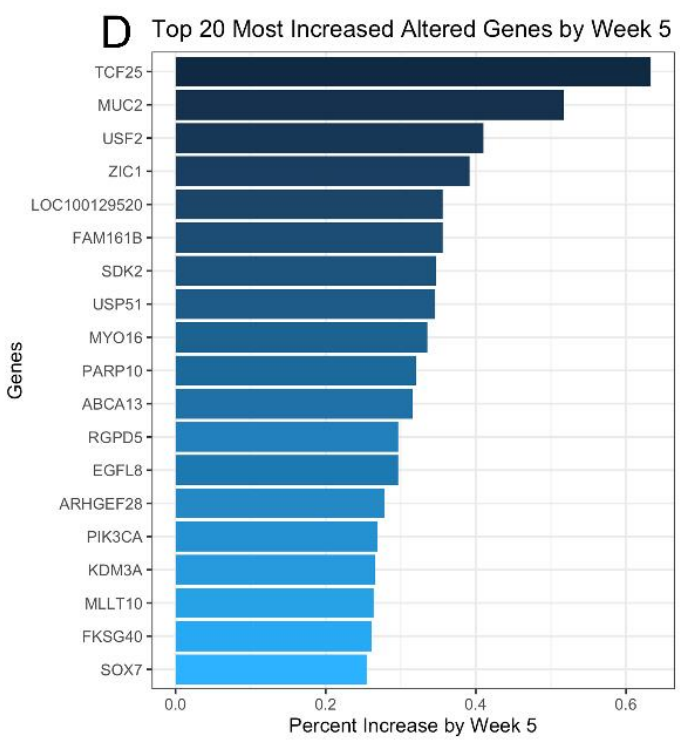

$E$

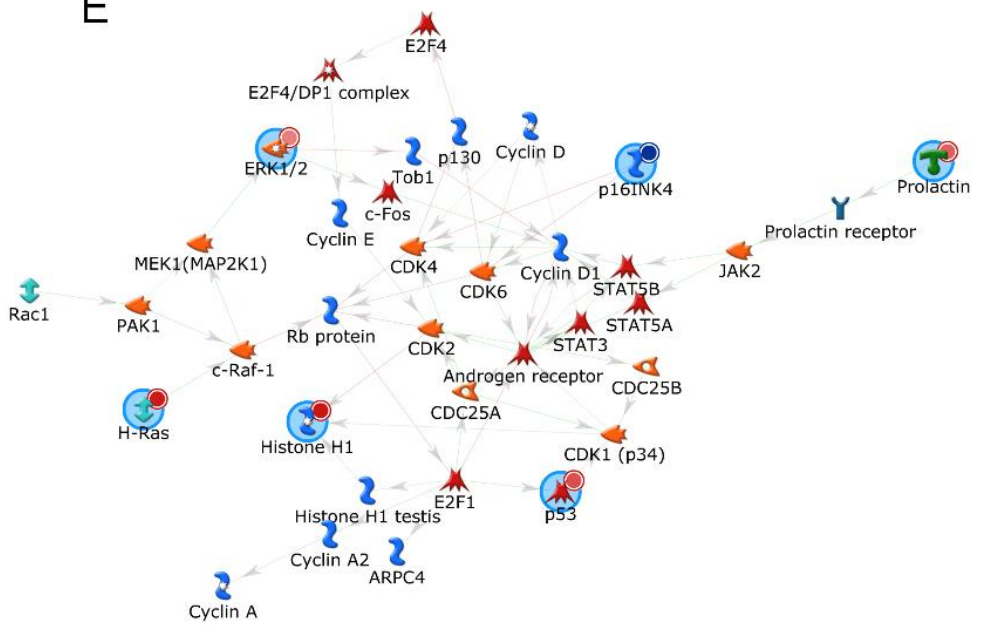

Figure 5. Individual mutations, insertions and deletions are highly dynamic throughout the course of CRT. (A) Demonstrates a representative poor responder with volume contoured to obtain change in overall tumor volume. (B) Demonstrates linear correlation between calculated tumor purity and MRI volume, used to adjust for relative mutation burden. (C) Ranked percent change in allele frequencies for individual genes summed by gene, demonstrating both increased and decreased allele frequencies from baseline to week 5. (D) Top 20 most increased altered genes by week5 in the overall population, suggesting potential drivers of radiation resistance. (E) The highest ranked pathway, according to increase over time and summed number of patients, centered on CDK4/CDK6/ and p16INK4 with included nodes highlighted in blue. 